\title{
COMMENT
}

\section{COVID-19 pandemic and its impact on mental health of healthcare professionals}

\author{
KONSTANTINOS TSAMAKIS ${ }^{1,2}$, EMMANOUIL RIZOS ${ }^{1}$, ATHANASIOS J. MANOLIS ${ }^{3}$, SOFIA CHAIDOU ${ }^{4}$, \\ STYLIANOS KYMPOUROPOULOS $^{1}$, ELEFTHERIOS SPARTALIS ${ }^{5}$, DEMETRIOS A. SPANDIDOS ${ }^{6}$, \\ DIMITRIOS TSIPTSIOS $^{7}$ and ANDREAS S. TRIANTAFYLLIS ${ }^{3}$
}

\author{
${ }^{1}$ Second Department of Psychiatry, University of Athens, 'ATTIKON' University Hospital, 12462 Athens, Greece; \\ ${ }^{2}$ King's College London, Institute of Psychiatry, Psychology and Neuroscience, London SE5 8AF, UK; \\ ${ }^{3}$ Department of Cardiology, Asklepeion General Hospital, 16673 Athens; ${ }^{4} 1$ st Department of Paediatrics, \\ University of Athens, Aghia Sophia Children's Hospital, 11527 Athens; ${ }^{5}$ 2nd Department of Propaedeutic Surgery, \\ National and Kapodistrian University of Athens School of Medicine, 11527 Athens; ${ }^{6}$ Laboratory of Clinical Virology, \\ School of Medicine, University of Crete, 71003 Heraklion; ${ }^{7}$ Neurology Department, \\ Evexia Rehabilitation Centre, 63080 Chalkidiki, Greece
}

Received April 3, 2020; Accepted April 7, 2020

DOI: $10.3892 /$ etm.2020.8646

\begin{abstract}
In light of the unprecedented public health crisis of the COVID-19 pandemic, it is highly important to acknowledge the psychological impact of this mounting threat on healthcare professionals. Previous experience from smaller scale epidemics and emerging literature around COVID-19 show that the unparalleled amount of stress that healthcare workers are dealing with, is associated with increased psychological morbidities. We have depicted the psychological burden that the COVID-19 pandemic has posed on healthcare professionals in Greece and have reviewed the literature around the effect of previous epidemics on frontline healthcare staff. Moreover, we discuss potential triggers and the need for measures to minimise the psychological pressure on those at the frontline against this biothreat.
\end{abstract}

\section{Introduction}

Since December 2019 the world has been facing an outbreak of a novel infectious disease known as Coronavirus disease (COVID-19), that has rapidly spread globally and has now been declared as a pandemic by the World Health Organisation (1).

Correspondence to: Dr Konstantinos Tsamakis, Second Department of Psychiatry, University of Athens, 'ATTIKON' University Hospital, Rimini 1, Chaidari, 12462 Athens, Greece

E-mail:ktsamakis@gmail.com

Key words: COVID-19, pandemic, mental health, anxiety, psychological impact, depression, healthcare professionals
Its spread and lethality is proving to be higher than previous epidemics on account of international travel density and immune naivety of the population (2), triggering urgent, draconian public health measures in many countries - Greece amongst them. This has caused an unprecedented situation for citizens, policy makers, politicians and healthcare professionals, with the pandemic being described as the worst public health crisis in a generation.

Psychological implications for general public, patients and children

Facing this largescale infectious threat, people are under increased psychological pressure. Data derived from previous epidemics, such as the Severe Acute Respiratory Syndrome (SARS) in 2003 and the H1N1 influenza in 2009, illustrate that the community suffered considerable fear and panic, resulting in a significant psychological impact $(3,4)$. This seems to be the case with COVID-19, as well. A very recent general public survey during the initial phase of the COVID-19 outbreak in China, where the epidemic began, showed that about one-third of the participants reported moderate-to-severe anxiety, whilst more than half rated the psychological impact as moderate-tosevere (5). Our personal experience in the outpatient clinics and wards in Greece coincides with the aforementioned report. During the past few weeks we have experienced a significant increase in telephone contacts and visits from patients or relatives expressing distress and fear about the virus and the surrounding uncertainty. Patients with mental health conditions, such as depression and anxiety have been reporting relapses in their mental state, such as fear-triggered panic attacks or resurface of psychosomatic symptoms; patients with medical comorbidities, such as cardiovascular disease, have been expressing distress 
and associated worsening of symptoms, in particular angina and worsening of heart failure status. Even though children have been reported to be less susceptible to COVID-19 infection (6), we have witnessed considerable psychological implications on them: the shutting down of schools and playgrounds, the restriction of outdoor activities alongside their parents' fears of contamination have been triggering manifestations of anxiety, such as panic attacks and psychosomatic symptoms.

\section{Psychological impact on healthcare professionals}

The general population, however, are not the only ones at risk for psychological distress during this pandemic. Experience from SARS and H1N1 epidemics underline that the psychological strain on healthcare professionals, who find themselves at the frontline of attempts to quell the outbreak, is significant $(3,4)$. In the early rapid expansion phase of the SARS outbreak, similar to the current course of COVID-19 pandemic, healthcare professionals reported feelings of extreme vulnerability, uncertainty and threat to life, alongside somatic and cognitive symptoms of anxiety (3), whilst during the 2009 H1N1 pandemic more than half of healthcare workers in a Greek tertiary hospital reported moderately high anxiety and subsequent psychological distress (4).

During the COVID-19 outbreak, clinicians in Greece have been confronted with mounting challenges that have not been faced before. Decisions have to be made fast, ranging from efficiently triaging and isolating patients with suspicion of infection, to deciding whether to shut down departments and operating theatres when a patient or staff test positive; all these whilst being on limited resources. The pressure to act timely and to successfully diagnose, isolate and treat has been overwhelming, especially amid intense public and media scrutiny. This is in concordance with experience in other countries (7). In addition, due to the increased risk of exposure to the virus, our frontline doctors, nurses and healthcare workers fear that they may contract COVID-19 themselves. They worry about bringing the virus home and passing it on to loved ones and family members - elderly parents, newborns and immunocompromised relatives. Our healthcare staff also report increased stress levels when dealing with patients unwilling to cooperate or not adhering to safety instructions, and feelings of helplessness when dealing with critically ill patients, in the context of limited intensive care beds and resources. The use of protective equipment for long periods causes difficulties in breathing and limited access to toilet and water, resulting in subsequent physical and mental fatigue. Very similar experiences of healthcare personnel have been recorded in the emerging scientific literature (8) and media reports in the two countries at present most heavily hit by COVID-19: China and Italy.

Therefore, healthcare professionals dealing with COVID-19 are under increased psychological pressure and experience high rates of psychiatric morbidity, resembling the situation during the SARS and H1N1 epidemics $(3,9)$. A very recent study among healthcare professionals in a tertiary infectious disease hospital for COVID-19 in China, revealed a high incidence of anxiety and stress disorders among frontline medical staff (10), with nurses having a higher incidence of anxiety than doctors. Experience in our hospitals in the past few weeks, although anecdotal, is concordant with these reports. The disruption of routine clinical practice, the sense of loss of control and the subsequent fear of potential destabilization of the health services, has provoked 'overflowing' anxiety and depression among healthcare professionals, a feature which is not uncommon of epidemics $(9,11)$. Depression is associated with poor medication adherence (12) which may increase morbidity among older healthcare professionals with coexisting medical conditions.

\section{Willingness of staff to work and their need for psychological support}

On a positive note, experience from our Departments so far, indicates that willingness of staff to work has not been really affected, in line with reports from previous pandemics (4). Despite the initial shock, the health professionals in the Greek National Health Service appear to exhibit high levels of commitment and professionalism. We know that confidence in safety, risk perception and confidence in skills are proven facilitators for willingness to work (11). Our impression is that the increasing knowledge about preventing and dealing with the disease, and the development of more specific procedural and treatment protocols, alongside educational activities, will contribute to improving the morale of healthcare workers dealing with the pandemic. However, in light of the increased psychological pressure of frontline healthcare staff, measures for psychological support and interventions to protect their mental health should be adopted promptly, as depicted from previous experience $(4,9)$ and emerging initiatives and literature $(7,8,13)$.

We are facing unprecedented circumstances; this biothreat is the most serious global crisis of our generation. Healthcare staff are in the frontline of this fight, which is taking a serious psychological toll; we therefore need to support them.

\section{Acknowledgements}

Not applicable.

\section{Funding}

No funding was received.

\section{Availability of data and materials}

The information generated and analyzed during the current study is available from the corresponding author on reasonable request.

\section{Authors' contributions}

KT wrote the original draft, edited and critically revised the manuscript. ER, ES, DAS, DT, SK, AJM, SC and AST critically revised and edited the manuscript. All authors substantially contributed to the conception, writing and revision of the work and approved the final content of the manuscript.

\section{Ethics approval and consent to participate}

Not applicable. 


\section{Patient consent for publication}

Not applicable.

\section{Competing interests}

The authors declare that they have no competing interests.

\section{References}

1. World Health Organisation: WHO announces COVID-19 outbreak a pandemic. http://www.euro.who.int/en/health-topics/ health-emergencies/coronavirus-covid-19/news/news/2020/3/ who-announces-covid-19-outbreak-a-pandemic. Accessed April 3, 2020.

2. Docea AO, Tsatsakis A, Albulescu D, Cristea O, Zlatian O, Vinceti M, Moschos SA, Tsoukalas D, Goumenou M, Drakoulis N, et al: A new threat from an old enemy: Re-emergence of coronavirus (Review). Int J Mol Med 45: 1631-1643, 2020.

3. Chong MY, Wang WC, Hsieh WC, Lee CY, Chiu NM, Yeh WC Huang OL, Wen JK and Chen CL: Psychological impact of severe acute respiratory syndrome on health workers in a tertiary hospital. Br J Psychiatry 185: 127-133, 2004.

4. Goulia P, Mantas C, Dimitroula D, Mantis D and Hyphantis T: General hospital staff worries, perceived sufficiency of information and associated psychological distress during the $\mathrm{A} / \mathrm{H} 1 \mathrm{~N} 1$ influenza pandemic. BMC Infect Dis 10: 322, 2010.

5. Wang C, Pan R, Wan X, Tan Y, Xu L, Ho CS and Ho RC: Immediate psychological responses and associated factors during the initial stage of the 2019 coronavirus disease (COVID-19) epidemic among the general population in China. Int J Environ Res Public Health 17: pii: E1729, 2020.
6. Lee PI, Hu YL, Chen PY, Huang YC and Hsueh PR: Are children less susceptible to COVID-19? J Microbiol Immunol Infect pii: S1684-1182(20)30039-6, 2020 [Epub ahead of print].

7. Greenbaum Z: Psychologist leads innovative approach to tackle psychological toll of COVID-19. Am Psychol Assoc. https://www. apa.org/news/apa/2020/03/psychologist-covid-19. Accessed March $15,2020$.

8. Chen Q, Liang M, Li Y, Guo J, Fei D, Wang L, He L, Sheng C, Cai Y, Li X, et al: Mental health care for medical staff in China during the COVID-19 outbreak. Lancet Psychiatry 7: e15-e16, 2020.

9. Brooks SK, Dunn R, Amlôt R, Rubin GJ and Greenberg N: A systematic, thematic review of social and occupational factors associated with psychological outcomes in healthcare employees during an infectious disease outbreak. J Occup Environ Med 60: 248-257, 2018

10. Huang JZ, Han MF, Luo TD, Ren AK and Zhou XP: Mental health survey of 230 medical staff in a tertiary infectious disease hospital for COVID-19. Zhonghua Lao Dong Wei Sheng Zhi Ye Bing Za Zhi 38: E001, 2020 [Epub ahead of print].

11. Aoyagi Y, Beck CR, Dingwall R and Nguyen-Van-Tam JS: Healthcare workers' willingness to work during an influenza pandemic: a systematic review and meta-analysis. Influenza Other Respir Viruses 9: 120-130, 2015.

12. Goldstein CM, Gathright EC and Garcia S: Relationship between depression and medication adherence in cardiovascular disease: the perfect challenge for the integrated care team. Patient Prefer Adherence 11: 547-559, 2017.

13. Vlessides M: COVID-19: Mental health pros come to the aid of frontline comrades. Medscape. $2020 \mathrm{https} / / / \mathrm{www} . m e d s c a p e . c o m /$ viewarticle/928004?nlid=134830_2052\&src=WNL_mdplsnews 200403 mscpedit_psyc\&uac $=216237 \mathrm{CR} \&$ spon $=12 \& \mathrm{impID}=23 \overline{3}$ 4311\&faf=1. Accessed April 3, 2020.

(i) () () This work is licensed under a Creative Common Attribution-NonCommercial-NoDerivatives 4.0 International (CC BY-NC-ND 4.0) License. 